\title{
Conflicto, territorio y extractivismo en Chile. Aportes y límites de la producción académica reciente
}

\section{Conflict, territory and extractivism in Chile. Contributions and limits of recent academic production ${ }^{1}$}

\author{
Antoine Maillet² (1), Mathilde Allain³ (1), Gonzalo Delamaza* (1),

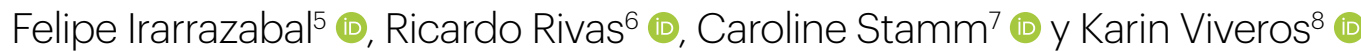

\begin{abstract}
RESUMEN
El presente artículo analiza la producción académica que aborda la problemática articulada por los conceptos de conflicto, territorio y extractivismo en Chile. El análisis de 32 artículos publicados en revistas indexadas entre 2015 y 2020 da cuenta de una preocupación importante por el fenómeno. Caracterizamos esta etapa de la literatura en relación al tratamiento de los distintos conceptos, así como de los métodos utilizados, los actores estudiados y las escalas consideradas. Esta producción académica aparece marcada por una rutinización, tanto en su despliegue conceptual, como en la predominancia de los estudios de caso. Para mantener el dinamismo de este campo de investigación, invitamos a complejizar la conceptualización de esta triada, diversificar los métodos y ampliar los actuales objetos de investigación.
\end{abstract}

Palabras clave: conflictos, territorio, extractivismo, escalas, Chile.

\begin{abstract}
This article analyzes the academic production that addresses the concepts of conflict, territory and extractivism in Chile. The analysis of 32 articles published in indexed journals between 2015 and 2020 accounts for a significant concern for this issue. We characterize this stage in this body of work in relation to its treatment of the different concepts, as well as the methods used, the actors studied, and the scales considered. This academic production appears marked by a routinization, both regarding the conceptual deployment and in the predominance of case studies. In order to maintain the dynamism of this field, we invite to take into consideration the theoretical depth of each concept, diversify the methods and broaden the objects of research.
\end{abstract}

Keywords: conflicts, territory, extractivism, scales, Chile.

Los autores agradecen el apoyo del Centro de Estudios de Conflicto y Cohesión Social COES (ANID/FONDAP/15130009), de Fondecyt (ANID /FONDECYT/1190070; ANID /FONDECYT/1180496; ANID /FONDECYT/ 11181188; ANID /FONDECYT/3190686), del Centro de Ciencia del Clima y la Resiliencia CR2 (ANID/FONDAP/15110009) y del Centro Nacional de Investigación para la Gestión Integrada de Desastres Naturales (ANID/FONDAP/15110017).

Instituto de Asuntos Públicos, Universidad de Chile. Investigador COES y CR2. Integrante del Colectivo de Estudios Político-Ambientales (CEPA). Correo electrónico: antoinemaillet@iap.uchile.cl

Instituto de altos estudios de América latina (IHEAL), Centro de investigación y de documentación sobre las Américas (CREDA), Paris Sorbonne Nouvelle. Investigadora COES. Correo electrónico: mathilde.allain@sorbonne-nouvelle.fr

CEDER Universidad de Los Lagos. Investigador COES. Correo electrónico: gonzalo.delamaza@ulagos.c

Instituto de Estudios Urbanos y Territoriales, Pontificia Universidad Católica de Chile. Investigador COES. Correo electrónico: firarrazava@uc.cl CEDER-Universidad de Los Lagos. Investigador COES. Correo electrónico: ricardo.rivas@ulagos.cl

Instituto de Estudios Urbanos y Territoriales, Pontificia Universidad Católica de Chile. Investigadora COES. Correo electrónico: castamm@uc.cl

Magíster en Ciencia Política de la Universidad de Chile. Correo electrónico: kviverosb@gmail.com 


\section{RESUMO}

Este artigo analisa a produção acadêmica que aborda os conceitos de conflito, território e extrativismo no Chile. A análise de 32 artigos publicados em revistas indexadas entre 2015 e 2020 mostra uma importante preocupação com o fenômeno. Caracterizamos esta etapa da literatura em relação ao tratamento dos diferentes conceitos, assim como os métodos utilizados, os atores estudados e as escalas consideradas. Esta produção acadêmica parece ser marcada por uma rotina, tanto em sua implantação conceitual como na predominância de estudos de caso. A fim de manter o dinamismo deste campo de pesquisa, convidamos a levar em conceituação a profundidade teórica de cada conceito, diversificar os métodos e ampliar os objetos atuais de pesquisa.

Palavras-chave: conflitos, território, extrativismo, escalas, Chile.

La promesa de alcanzar el desarrollo mediante la extracción de recursos naturales ha sido un tema controversial y debatido recurrentemente en la historia de América Latina (Cardoso \& Faletto, 1969; Prébisch, 1950). Sin embargo, el auge en la extracción de recursos naturales que ha experimentado la región en los últimos 20 años, en el período llamado superciclo, reconfiguró las relaciones espaciales entre los lugares de extracción, las áreas metropolitanas y la economía global (Arboleda, 2020; Bebbington, 2009). Una de las principales características de esta reconfiguración ha sido el surgimiento masivo de conflictos territoriales desde la escala local. Ello no solamente refleja las tensiones entre comunidades locales y elites nacionales, que articulan estrategias de acumulación en base a la extracción de recursos naturales (Arsel et al., 2016; Bebbington \& Bury, 2014; Martínez-Alier, 2015), sino también diferentes aspiraciones de desarrollo y formas de relación con la naturaleza que existen en la región (Alimonda et al., 2017; Escobar, 2008; Porto-Gonçalves \& Leff, 2015).

Estas tensiones entre los modelos de desarrollo basados en la extracción de recursos naturales y los proyectos locales que buscan formas alternativas de gobernanza han sido discutidas por las ciencias sociales. Un hito clave al respecto, el cual posicionó los términos de 'extractivismo' y 'neoextractivismo' en el debate, fue la publicación de libro 'Mas allá del desarrollo' por parte del Grupo Permanente de Trabajo sobre Alternativas al Desarrollo del Consejo Latinoamericano de Ciencias Sociales (CLACSO). En ese libro se encuentran los influyentes trabajos de Maristella Svampa (2012), Alberto Acosta (2012) y Eduardo Gudynas (2012), los cuales comenzaron a caracterizar las aristas que definen la actual fase de desarrollo basado en la extracción de recursos naturales en la región. En ese contexto, el concepto de 'extractivismo' se refiere fundamentalmente al modo actual de acumulación de las economías latinoamericanas, el cual se estructura sobre la apropiación masiva de recursos naturales, y su exportación con escaso procesamiento a mercados internacionales (Svampa, 2019). El neoextractivismo a su vez se refiere a cómo ciertos gobiernos de izquierda mantuvieron el enfoque extractivista y adoptaron esta práctica durante el denominado "commodity boom" en los años 2000, financiando así sus políticas sociales.

La relevancia de la minería del cobre en la economía nacional y las múltiples resistencias locales en torno a esta actividad, así como a la extracción de otros minerales (oro, litio), la industria forestal, la salmonicultura, entre otras, posicionan a Chile como un caso relevante para estudiar las contradicciones entre el modelo de acumulación y los proyectos y modos de vida de los actores locales (Bolados, 2016; Fernández, 2020; Romero-Toledo, 2019; Akchurin, 2020). El coloquio permanente sobre Conflictos Territoriales al alero del Centro de Estudios sobre Conflicto y Cohesión Social (COES) ha buscado desde el año 2018 crear un espacio de convergencia académica 
para quienes han estado investigando y conceptualizando los conflictos territoriales como una expresión de la interfaz entre lo local y lo global. En este espacio han participado expositoras y expositores de distintas disciplinas y partes del mundo, quienes han dado cuenta de avances y resultados de investigación respecto al territorio como un escenario de disputas entre grupos locales, las elites estatales y el capital global, principalmente representado por industrias extractivas y proyectos energéticos.

Para dar continuidad a este proceso colectivo consideramos necesaria una revisión sistemática de la producción existente en torno a los términos de conflicto, territorio y extractivismo en Chile, como un ejercicio reflexivo respecto a la articulación conceptual en la variopinta producción académica en la materia ${ }^{9}$. En general, en la línea de contribuciones emblemáticas como el giro eco territorial (Svampa, 2012), se tiende a dar por sentada la articulación conceptual entre estos tres términos. Sin embargo, es importante conocer la forma en la cual se han usado estos conceptos, y las relaciones establecidas entre ellos en las investigaciones. De esta forma, podremos analizar la manera en la cual se ha desplegado en Chile la reflexión relacionada con la intensificación del extractivismo y de la difusión de marcos de análisis latinoamericanos. Si bien diversos enfoques disciplinares e interdisciplinares han dado aproximaciones genéricas a problemáticas similares, estás no fueron consideradas para enfocarnos de manera más precisa en el uso de los tres conceptos en cuestión. Este es el caso de la Ecología Política, la cual ha abordado críticamente la gobernanza ambiental del país mediante conceptos como poder, naturaleza y propiedad (Bustos et al., 2015), pero cuenta todavía con pocas publicaciones sobre el tema del extractivismo, a diferencia de los conflictos ambientales y del territorio (Bustos \& Prieto, 2019), o bien el cuantioso y valioso trabajo de activistas y ONGs en torno a conflictos ambientales.

Preguntas de nuestro interés para esta descripción son si existe un uso común de estos conceptos, qué metodologías se han utilizado para llevarlos al estudio empírico, y si permiten dar cuenta de la conflictividad extractivo-territorial en Chile en su complejidad. También, nos interesa evaluar el potencial de estas investigaciones para dar cuenta de nuevas tendencias que están apareciendo en el fenómeno en sí, como la multiescalaridad y la diversificación de los actores participantes en estos conflictos. Este ejercicio es fundamental para el trabajo interdisciplinario, no solo por un tema de precisión conceptual, sino también para proyectar un mejor entendimiento respecto a las dinámicas espaciales, sociales y políticas del extractivismo.

El presente artículo muestra una revisión sistemática de la literatura académica reciente sobre extractivismo, conflictos y territorio en Chile, conformada por 32 artículos publicados en revistas con indexaciones Wos, Scopus y Scielo durante los últimos 5 años. La misma aplicación de los criterios de búsqueda limitó el alcance temporal del estudio. El argumento central que se propone es que esta producción académica es principalmente convergente, con poco debate y controversias, a diferencia de los estudios latinoamericanos sobre extractivismo, donde se han producido debates que dan cuenta de algunas divergencias. Por ejemplo, en una primera instancia se dieron tensiones respecto a la posibilidad de generar proyectos transformadores o posneoliberales a partir de la renta extractiva (Svampa \& Stefanoni 2007; Gudynas 2010), lo cual derivó en la literatura sobre 'Neo extractivismo'. Otro ejemplo fueron las críticas que planteó Eduardo Gudynas

En este sentido, este trabajo no pretende entregar una definición acabada de los distintos conceptos, sino dar cuenta de la variedad - y a veces indefinición - en su uso. 
a quienes usaban literaturas anglófonas, fundamentalmente la 'acumulación por desposesión' de David Harvey, para abordar los conflictos en torno a industrias extractivas. Esta crítica, la que Gudynas sintetizó con la frase 'terminar con el colonialismo simpático', fue respondida por el equipo del Centro Nacional de Estrategia para el Derecho al Territorio (CENEDET) en Ecuador, el cual era cercano a Harvey, y contó con intervenciones de destacados autores como Joan Martínez Alier. Si bien estas discusiones tuvieron un eco en la literatura sobre extractivismo a nivel internacional, tuvieron escasa incidencia en la literatura académica sobre Chile.

Esto nos lleva a observar una tendencia a la rutinización de la investigación, que se manifiesta en el uso extendido de ciertos conceptos, pero sin la suficiente profundización y cuestionamiento teóricos. Comprendemos rutinización desde la perspectiva de Berger \& Luckmann (1966), quienes buscando sentar las bases de una Sociología del Conocimiento, analizan el proceso de rutinización que facilita la institucionalización de las interacciones sociales. No se trataría solo de una regularidad de comportamientos interactivos, sino lo que cognitivamente se rutiniza, lo que se da por hecho (taken-for-grantedness), cierto u obvio respecto a un objeto, práctica u organización sin mayor proceso reflexivo. Por consiguiente, el sentido y significado de ciertos conceptos como los que analizamos en este trabajo se "da por sentado" ante una comunidad epistémica asumida, lo cual facilita la comunicación, pero limita la elaboración conceptual necesaria a un enfoque reciente. En el mismo sentido argumenta Skilton (2006) y otros autores anglosajones. Otro aspecto importante es la predominancia metodológica de los estudios de caso, en los que se tiende a situar espontáneamente los casos de estudio dentro de la categoría del extractivismo e interpretar en ese marco los conflictos, sin explotar realmente todas las potencialidades de este marco conceptual. Si bien la investigación social basada en caso único o solo algunos casos ha sido considerada una alternativa necesaria en la construcción y validación teórica (Ragin 1992; Yin 2009), es necesario que este tipo de investigación no descuide la precisión conceptual tanto en los antecedentes teóricos de un eventual proceso deductivo como en su etapa inductiva de generación teórica.

El artículo se organiza de la siguiente manera. Primero detallamos la metodología de constitución del corpus revisado y los análisis aplicados, que nos permiten realizar un primer análisis descriptivo de las principales características de esta producción académica. Luego, el análisis transversal del uso de los tres términos de interés - conflicto, extractivismo, territorio - y de tres temáticas - metodología, escalas y actores - nos conduce a calificar el campo de estudio como rutinizado. En la última sección, indicamos algunas pistas para profundizar el estudio del fenómeno, con base en la renovación de las metodologías, la revisión de la aplicación de los conceptos y la apertura hacia nuevas temáticas, que permitan hacer sentido de nuevas tendencias y la intensificación del extractivismo chileno y latinoamericano.

\section{Metodología}

Con el objetivo de conocer el estado del campo de investigaciones sobre los conflictos extractivo-territoriales en el contexto chileno, llevamos a cabo una estrategia de análisis de contenido que se define como un conjunto de procedimientos con el fin de obtener inferencias válidas de dichos textos (Weber 1990). Específicamente nuestra técnica se basa en un análisis semántico de artículos científicos considerando criterios de confiabilidad y validez de dicha técnica (Krippen- 
dorff 2003). Respecto a la confiabilidad, el resultado de los criterios de clasificación es estable, reproducible y preciso después de diversas búsquedas en distintas ocasiones usando los mismos criterios. Respecto a la validez interna, se trata de una validez semántica de las tres palabras clave de búsqueda que se cauteló a través de la lectura de los resúmenes de cada uno de los artículos contemplados en una primera ronda de búsqueda.

En términos procedimentales, iniciamos un proceso de búsqueda sistemática de artículos en torno a tres conceptos: conflicto, extractivismo y territorio ${ }^{10}$. Uno de los criterios de selección fue considerar sólo artículos en revistas académicas donde aparecieran estas tres palabras más la palabra Chile en los apartados del título, resumen y/o palabras claves"1, utilizando los operadores booleanos "AND" y "OR" para la búsqueda. Al respecto, para incrementar la recuperación de trabajos en este campo de estudio, se consideraron algunas variantes de los conceptos señalados, para lo cual, se aplicó en las palabras de búsqueda el comando $\left({ }^{*}\right)$, lo que permitió ampliar el universo a todas las palabras que inicien con estos términos, incluyendo, por ejemplo, los conceptos en singular como en plural (Ver Cuadro 1).

Cuadro $\mathrm{N}^{\circ} 1$.

Proceso de búsqueda en repositorios académicos y resultados.

\begin{tabular}{|c|c|c|}
\hline SCIELO & IOS & JS \\
\hline $\begin{array}{l}\left.\text { (conflict*) AND } \text { (extractiv }^{*}\right) \\
\text { AND (chile*) AND (territori* } \\
\text { OR ambient* OR socio-eco- } \\
\text { logic* OR socio-ambient* OR }^{*} \text { socioambient* OR socioeco- }^{*} \text { OR } \\
\text { logic* OR socioterritori }{ }^{*} \text { OR } \\
\text { socio-territori*) en "Todos los } \\
\text { indices" }\end{array}$ & $\begin{array}{l}\text { (conflict*) AND (chile* AND }^{*} \text { AN }{ }^{*} \text { territor* OR environment* OR } \\
\text { socioterritorial* OR socioen- } \\
\text { vironmental* OR socio-envi- } \\
\text { ronm* OR socio-territorial* OR }_{\text {socioecologic }}^{*} \text { OR socio-eco- } \\
\text { logic*) AND (extractiv*) en 'Te- } \\
\text { mas' }^{\prime}\end{array}$ & $\begin{array}{l}\text { (conflict* AND chile* AND ex- } \\
\text { tractiv* AND (territor* OR so- }^{*} \text { cioterritor* OR socio-territor* }^{*} \\
\text { OR environment* OR socio-en- } \\
\text { vironmen* OR socioenviron- } \\
\text { men* OR socio-ecolog* OR } \\
\text { socioecolog*)) en 'Título, abs- } \\
\text { tract y Keywords' }\end{array}$ \\
\hline $\begin{array}{l}\text { e búsqueda= } 9 \\
\text { e cumplen los cri- } \\
\text { squeda }=8\end{array}$ & $\begin{array}{l}\text { Resultados de búsqueda }=23 \\
\text { Artículos que cumplen los cri- } \\
\text { terios de búsqueda }=20\end{array}$ & $\begin{array}{l}\text { Resultado de búsqueda=29 } \\
\text { Artículos que cumplen los cri- } \\
\text { terios de búsqueda }=23\end{array}$ \\
\hline
\end{tabular}

Fuente: Elaboración propia.

Como mencionamos, dentro de los criterios de selección consideramos sólo aquellas investigaciones acerca de Chile, o sobre algún territorio dentro del país, donde se aborden en el estudio los conflictos territoriales o socioambientales ${ }^{12}$. Esto implicó que, con posterioridad a la búsque-

Para la revisión de este concepto se añadieron otras categorías de búsqueda que permitieron ahondar el abordaje del territorio y la dimensión territorial en el estudio de los conflictos relacionado al extractivismo, incluyendo así distintos tipos de conflictos, como conflictos territoriales, ambientales, socioambientales, socio-ecológicos y socio-territoriales (ver Cuadro 1). Estos conceptos diversos fueron considerados de forma amplia ya que refieren a elementos comunes, tales como la disputa por el territorio, enfrentamiento de distintas territorialidades o la gestión de los bienes comunes, lo que permite incluir en la revisión distintos enfoques de lo territorial en los trabajos sobre conflictos y extractivismo.

1 Dentro de las palabras claves se incluyen las palabras claves asignadas por los mismos repositorios de forma automática para facilitar los procesos de búsqueda.

12 Para ello, definimos utilizar Chile (chile* para incluir otros conceptos como región chilena) como motor de búsqueda ya que el objetivo fue recoger artículos que hablen explícitamente del país, ya sea a nivel nacional u otra escala. Si bien, se considera que algunas de las plataformas empleadas tienen la opción de filtrar la búsqueda por país, la diferencia entre los resultados de ambas vías se corroboró que es mínima. 
da, en los repositorios académicos señalados, se aplicaron filtros de pertinencia sobre el corpus final. De los 61 artículos encontrados inicialmente, se restaron aquellos 10 artículos que no cumplían los criterios de búsqueda y aquellos 19 artículos que se encontraron en más de una de estas bases de datos. De esta forma, se procesaron 61 resultados en los repositorios, de los cuales 32 artículos cumplieron con los criterios de selección durante el periodo de consulta, desarrollado a inicios de diciembre de $2020^{13}$. Cabe señalar, que el periodo de búsqueda ni el idioma fueron restringidos o se utilizaron como filtro de los artículos.

Para la revisión utilizamos las bases de datos de Web Of Science (WOS), Scopus y Scielo, ya que esta se centró exclusivamente en artículos académicos. Al respecto, es importante señalar que una gran cantidad de trabajos y valiosos aportes se han desarrollado desde organizaciones sociales y ONGs, como por ejemplo Chile Sustentable, OCMAL, OLCA o MODATIMA, en torno a este tema. Sin embargo, estos no fueron considerados dentro de este corpus, centrado sólo en trabajos académicos, no incluyendo tampoco en la muestra capítulos de libros, libros, conferencias e informes. Por ende, cabe señalar que las publicaciones encontradas y revisadas no constituyen la totalidad de los trabajos sobre conflictos territoriales y extractivismo en Chile o de investigaciones que hagan referencia a estos conceptos y fenómenos. Eso configura un corpus significativo sobre el que establecer algunas reflexiones en torno a los enfoques empleados y la evolución del campo de investigación en esta materia. El enfoque que nos interesa es la discusión teórica y conceptual por lo cual nos enfocamos en la producción académica, la cual se articula de todas maneras con otras producciones. No cabe duda que las articulaciones entre las diferentes producciones y entre academia y mundo de la movilización son muy importantes.

Finalmente, queremos destacar que nuestra búsqueda inicial no implicaba una restricción en el período abarcado. Es el resultado arrojado por la combinación de los criterios de búsqueda que llevó a reunir artículos de un período reciente, dejando así claro que se trata de una discusión todavía emergente.

\section{Análisis descriptivo del corpus}

Desarrollamos un levantamiento sobre datos descriptivos de las investigaciones, en base a información sobre los artículos y sus autores y autoras. En cuanto a las investigaciones, estas se distribuyen dentro del periodo de 2015 a 2020, concentrándose, principalmente, durante los dos últimos años, con más de la mitad de los artículos publicados en 2019 o 2020. Por otro lado, las publicaciones se dividen equitativamente entre artículos en español e inglés, incluyendo en el corpus sólo un trabajo en otro idioma, el artículo de Bolados \& Boccara (2015) en francés. Esta limitación temporal y lingüistica del corpus es importante de tomar en cuenta para matizar nuestras propias conclusiones. Es decir, puede tratarse de un estadio muy temprano de desarrollo del campo, el cual todavía se mueve en un cierto "boom" teórico y dentro de una comunidad lingüística. No podemos predecir el comportamiento futuro del campo, que pudiere variar según se amplíe.

Al no aplicar un periodo de tiempo determinado a la búsqueda, el criterio temporal obedece a la presencia de los artículos respectivos en los repositorios académicos en el periodo de búsqueda (concluido a principios de diciembre de 2020), por lo cual, es posible puedan variar los números de la muestra con posterioridad a la inclusión de nuevas publicaciones que cumplan estos criterios de búsqueda. 
En cuanto a los sectores extractivos que abordan los trabajos, casi la mitad (15 artículos) se centran en la minería, seguido de 8 trabajos sobre proyectos energéticos, en su mayoría del sector hidroeléctrico, 5 de los trabajos son sobre la industria forestal, otros 5 sobre el sector agroexportador y 2 sobre la industria acuícola. Cabe señalar que hay trabajos que abordan en su estudio más de una industria extractiva, así como hay otros que no se enfocan en ninguna en particular, estos casos involucran a 5 investigaciones del corpus.

Respecto a la ubicación geográfica de los casos estudiados, 9 artículos no se ubican en una región político-administrativa en particular o un territorio dentro de alguna. Respecto a aquellos casos en que, si se aborda una región o un territorio determinado dentro de alguna, la región de Valparaíso es la región más analizada, le siguen la región de Tarapacá y la región del Biobío (Ver Figura 1). De forma particular, se destacan el territorio denominado como Wallmapu y/o Gülumapu, que, si bien no es considerado dentro de la división político-administrativa, es un espacio reconocido dentro de 3 artículos. Aunque no delimitado en estos, es caracterizado como el territorio ancestral mapuche y los estudios de campos al respecto se emplazan dentro de las regiones del Biobío y la Araucanía.

Figura $\mathrm{N}^{\circ} 1$.

Cantidad de artículos según la región sobre el que se desarrolla la investigación.

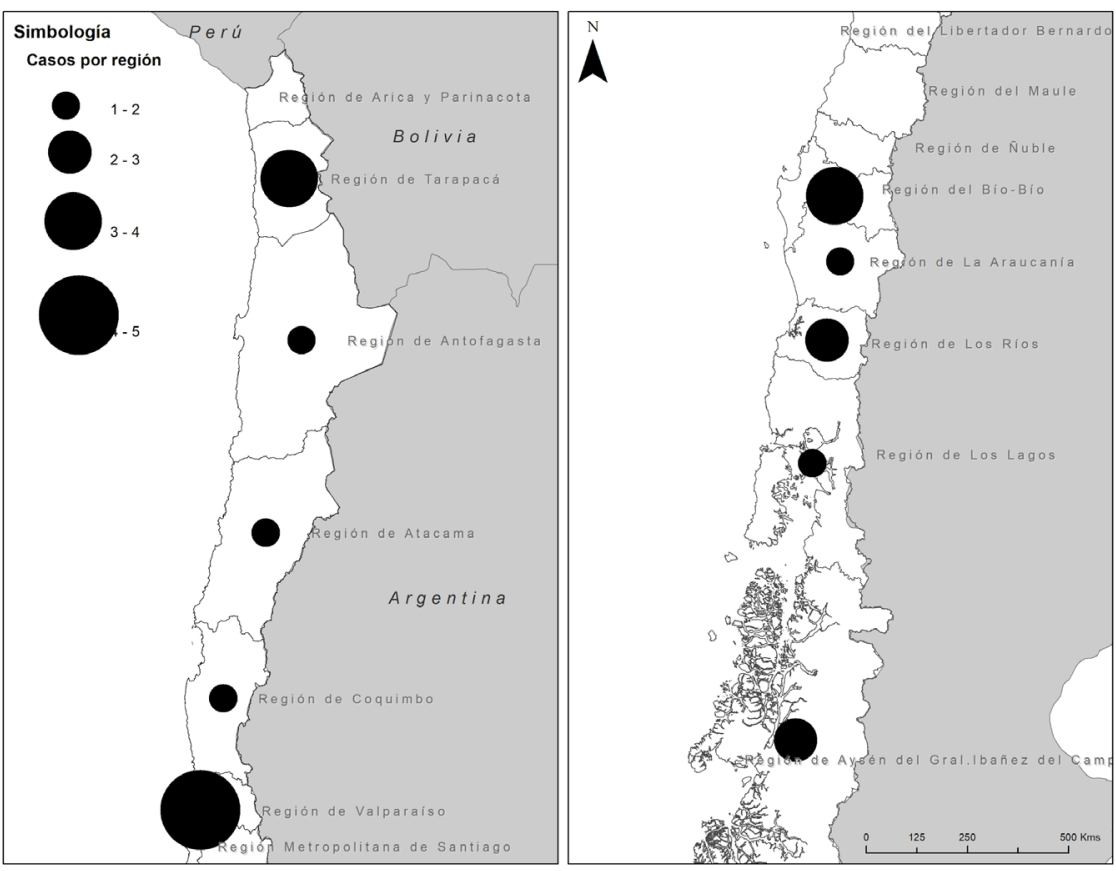

Fuente: Elaboración propia.

En cuanto a la autoría de los artículos del corpus, 38 autores son hombres y 29 son mujeres. Respecto a su afiliación institucional ${ }^{14}$, de 67 personas, 48 de los autores/as pertenecen a una ins-

14 Según la afiliación indicada al momento de publicar las investigaciones. 
titución ubicada en Chile, de estas, 17 se encuentran en la Región Metropolitana y las otras 32 son de instituciones de diversas regiones del país (Ver Figura 2). Por otro lado, 17 personas son parte de instituciones ubicadas en el extranjero: 7 personas pertenecientes a instituciones de España, 3 de Reino Unido, 2 de Argentina, 2 de Estados Unidos y otras 6 personas de diferentes instituciones en otros países de Europa, Oceanía y América Latina.

Figura $\mathrm{N}^{\circ} 2$.

Autoras y autores del corpus, según su afiliación institucional.

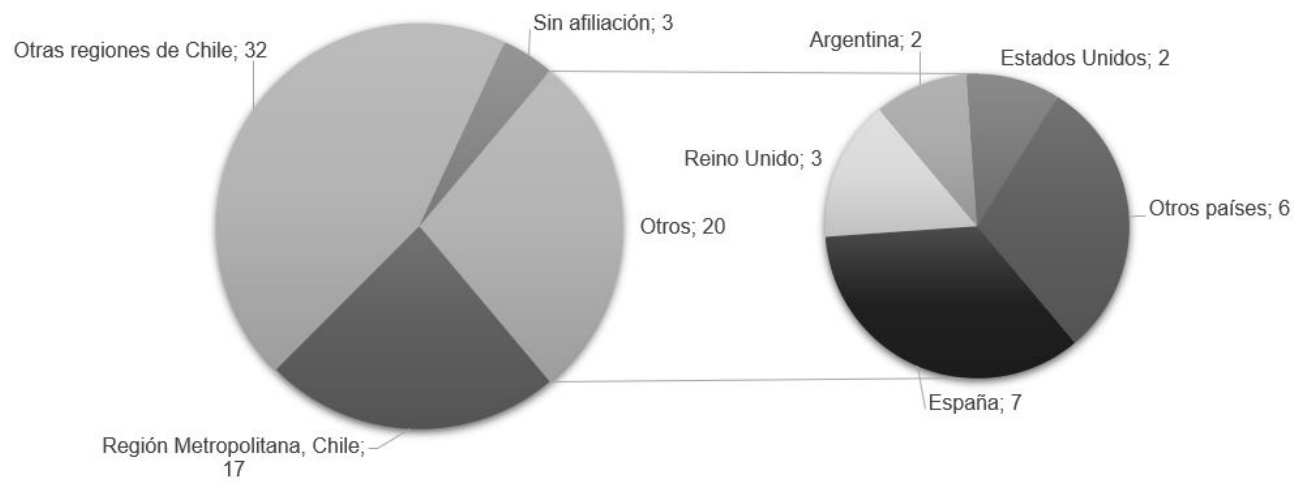

Fuente: Elaboración propia.

\section{Una campo convergente ¿rutinización de la investigación?}

De acuerdo con los criterios de selección de los artículos del corpus, analizamos de manera transversal cómo se abordan y definen los conceptos de conflicto, extractivismo y territorio en los textos, para caracterizar el campo de investigación. Luego, para ir más allá de los conceptos, buscamos entender cómo se ha desarrollado este campo y qué estrategias han adoptado sus autores, por lo cual nos enfocamos en 3 dimensiones: los actores estudiados, la(s) escala(s) de análisis y la metodología de investigación.

El análisis del corpus de artículos revela el desarrollo relevante de investigaciones que retoman el concepto de extractivismo en el caso chileno y analizan los conflictos relacionados con éste en distintas regiones a lo largo del país. Son principalmente estudios de casos que permiten describir varios tipos de conflictos en distintos sectores extractivistas, y que revelan procesos similares desde una escala ante todo local. Como lo discutimos más en detalle en los párrafos siguientes, respecto al uso de los conceptos de conflicto, extractivismo y territorio, se puede observar que se aplican generalmente sin definirlos en profundidad y sin generar en definitiva un desarrollo teórico sobre ellos a partir del caso chileno. Las estrategias de investigación convergen en el estudio cualitativo de casos en su mayoría, privilegiando el estudio de caso único. Respecto a los actores, se tiende a abordar la triada clásica Estado-Empresa-Comunidad, con la elección de una escala única, principalmente local, y fija. Planteamos entonces la hipótesis de una rutinización de la investigación sobre conflictos, extractivismo y territorios, que vamos a revisar a continuación. 


\section{Conflicto: visiones convergentes}

El concepto de conflicto es central en las investigaciones revisadas, principalmente, en su expresión como conflicto territorial o socio-ambiental, aunque son pocos los trabajos que se detienen a desarrollar lo que entienden por conflicto, sino que se asume que el concepto se entiende en su sentido general y común, sin incluir una dimensión teórica y crítica en su uso.

El acercamiento al conflicto en el corpus de artículos se caracteriza por cuatro elementos convergentes. Primero, el conflicto se asume en su dimensión abierta, es decir que los trabajos se enfocan en los conflictos en los cuales hay oposiciones que se traducen en el espacio público, dejando de lado los conflictos latentes o poco visibles. El estudio del conflicto desde la perspectiva de la acción colectiva tiene, en ese sentido, relevancia en el corpus. Segundo, se entiende que el conflicto se desarrolla entre grupos sociales con objetivos incompatibles, tendiendo a polarizar el conflicto entre los que dan origen al conflicto, a través de su intervención, y los que resisten esta transformación. Esto deja en segundo plano un matiz importante de posiciones dentro del conflicto, el proceso de constitución y transformación de actores durante el conflicto y un análisis de la diversidad de actores. Tercero, el conflicto es, en este sentido, mayoritariamente visto como un proceso esperable, ilustrando procesos de resistencia de parte de los actores locales. De hecho, en distintos trabajos, se reemplaza el conflicto por los términos de resistencia o lucha. Cuarto, en relación con la tendencia anterior, el conflicto es asumido en general como una situación asimétrica entre los actores involucrados.

Esta convergencia se ilustra en algunas definiciones encontradas, las cuales abordan el conflicto como resultado de la desavenencia entre actores, las que se anclan en diferencias valóricas y de objetivos entre grupos sociales (Ocampo et al, 2018), como situación en las cuales dos o más actores persiguen objetivos mutuamente incompatibles (Heinelt, 2019), como disputa entre grupos sociales respecto a la apropiación de los recursos naturales o el territorio (Santibáñez, 2018) o como movilizaciones físicas de poblaciones vecinas que se enfocan en el impacto negativo percibido de una industria (González \& Zografos, 2017). En su dimensión socioambiental, el conflicto se entiende como una situación surgida a partir del impacto ambiental producido por una determinada acción, en torno a la cual se enfrentan tres tipos de actores: empresas que usan recursos ambientales y generan un impacto, la comunidad organizada en torno a la defensa de sus intereses y agencias públicas con responsabilidades de mediación en el conflicto (Fernández, 2020).

\section{El uso rutinizado del extractivismo}

Respecto al concepto de extractivismo, la mayoría de los trabajos usa el concepto, principalmente, como adjetivo, por ejemplo, como "mentalidad extractivista", "imperativo extractivista", "lógica extractivista" o "paradigma extractivista". Incluso está la incorporación de la referencia en plural como "extractivismos" para señalar la existencia de ciertos "estilos" dependiendo del tipo de la rama económica y los territorios involucrados (Bolados et al., 2017). Teniendo en cuenta estas variantes, el concepto tiene una función de contexto para el análisis de cada objeto de estudio, pero sin profundizar en una perspectiva o definición específica. Se asume entre los autores y autoras una connotación compartida haciendo innecesario mayor despliegue analítico-conceptual. Es lo que hemos detectado como una rutinización o inercia conceptual que suspende el 
cuestionamiento teórico independiente del poder comunicativo del concepto. Es lo que en la literatura anglosajona se ha conocido como el "taken-for-grantedness" de comunidades científicas (Skilton, 2006).

Sin embargo, hay excepciones puntuales (Fernández, 2020; Pino \& Carrasco, 2019; Mancilla, 2017; Cuevas \& Budrovich, 2020) que entregan definiciones que tienen como centro los procesos de extracción y explotación de recursos naturales destinados a la exportación y a la inserción en el mercado global (Bolados et al., 2017). Dentro de estos esfuerzos de definición, se da una distinción interesante entre extractivismo y "neoextractivismo". Si bien este concepto surge en relación con ciertos gobiernos en América Latina que basaron su gasto social en el denominado "commodity boom" en los años 2000, ha comenzado a significar otros casos nacionales donde el Estado ha adquirido mayor protagonismo en el control y redistribución de los recursos provenientes de la actividad extractiva, principalmente, la minería. Siguiendo el trabajo de Maristella Svampa, estaríamos en un nuevo ciclo de este modelo asociado al mercado financiero global (Mancilla, 2017; Reyes \& Rodríguez, 2015; Bolados \& Sánchez, 2017). El caso chileno, si bien no es un ejemplo ampliamente recurrido para analizar este fenómeno, presenta características en cuanto a nuevas instituciones e imaginarios en torno a su denominada "segunda fase de exportación" heredada de las medidas neoliberales respecto a una estrategia comercial internacional desde los años ochenta. Es así, como el cultivo de frutas (uvas), árboles (pino y eucaliptus) y peces (salmones) se convierten en una alternativa al extractivismo de raigambre colonial, pero produciendo similares secuencias socioecológicas a diferentes escalas geográficas.

Finalmente, otro aspecto interesante que podemos notar es que el concepto de extractivismo no sólo denota procesos físicos o tangibles asociados a ciertas actividades económicas, sino también en estrecha conexión con sus consecuencias socioculturales o simbólicas en la construcción de espacios (Romero-Toledo, 2019) y en la profundización de un proyecto culturalmente colonizador (González et al., 2019).

\section{La simplificación del territorio}

En cuanto al aspecto territorial de los conflictos, muy pocos son los trabajos que abordan o definen qué se entiende por territorio en el marco de la relación con conflicto y extractivismo. En la mayoría del corpus, el territorio se asimila al territorio político-administrativo o se considera simplemente como el soporte físico del conflicto, siendo casi sinónimo del espacio local, pero sin caracterizarlo y entenderlo como una construcción social. Cuando se usa el término de conflictos territoriales, se reduce el territorio a un adjetivo, que parece indicar que el conflicto ocurre en un territorio y para este territorio, sin generalmente ir más allá en la conceptualización. Eso impide abordar el territorio como una producción dentro de los conflictos socio-ambientales, con múltiples dimensiones más allá de la física.

Sin embargo, algunas investigaciones ponen atención al concepto de territorio, particularmente desde el campo de la geografía, como lo demuestran las definiciones siguientes extraídas del corpus de artículos. Para Rodríguez et al. (2020:2904), el territorio es "entendido como espacio vivido, y como apropiación simbólica vivencial de dimensiones históricas, sociales y culturales recuperadas a través de la memoria dentro de los repertorios de acción colectiva" a lo que añade, citando a Escobar (2015), "los territorios se convierten simultáneamente en 'espacio-tiempos', 
tanto de la comunidad y de interrelación de lo que circunda la experiencia" (Rodríguez et al., 2020:2914). En el texto de Fernández (2020), el territorio es un espacio social y político, que se construye a partir de las relaciones sociales que ocurren entre los actores. A partir de la definición de Porto-Gonçalvez (2009), lo define como "un espacio social que es apropiado y significado por sujetos y grupos que se afirman por medio de él, y en el cual hay relaciones sociales en tensión y diversas territorialidades" (Fernández, 2020:226). Romero-Toledo ha analizado y desarrollado el concepto, desde la geografía, definiéndolo como el proceso en el cual la naturaleza empezó a ser socialmente producida. Teoriza el territorio como "el resultado de la tensión entre la reproducción capitalista y los espacios cotidianos, afectivos y esenciales de los grupos locales y comunidades indígenas"15 (Romero-Toledo, 2019:9). En otro texto co-escrito con Sambolin sobre indigeneidad y territorio, describe este último como espacio, poder, significado y experiencia. Más que espacio geopolítico del Estado o el espacio delimitado por las fronteras del Estado, es un espacio relacional que se construye por diferentes actores a través de prácticas cotidianas, de estrategias de negociación y resistencia, así como de relaciones de poder localizadas, pero articuladas con lo global (Romero-Toledo \& Sambolin, 2019). Por último, desde la ecología política, González \& Zografos (2017) proponen otra definición en su estudio sobre el rol de las emociones en conflictos forestales, identificando el territorio como una forma de pensamiento político y una forma de sentimiento político en términos físicos, emocionales y espirituales.

Cabe también destacar aquí la referencia, en varios artículos, al giro eco-territorial descrito por Svampa (2012: 22), como "la emergencia de un lenguaje común que da cuenta del cruce innovador entre la matriz indígena comunitaria, la defensa del territorio y el discurso ambientalista". Ilustra la gran recepción en Chile de la reflexión sobre extractivismo lanzada por CLACSO, y su integración en el corpus de trabajos chilenos.

\section{Los actores analizados}

En cuanto a los actores que están implicados directa e indirectamente en situaciones de conflicto en los casos investigados, la mayoría de los trabajos considera un espectro amplio (Rodríguez, et al., 2020; Silva, 2016; Palma \& Alcaino, 2020). Una minoría se centra solo en dos actores principales, como es el caso de conflictos entre comunidades indígenas y otro actor en oposición (Bolados \& Boccara, 2015; González \& Zografos, 2017). Podemos afirmar que los estudios con múltiples actores consideran en general la triada Estado-Empresas-Sociedad Civil. En cuanto al Estado, se trata más bien de agencias gubernamentales de carácter central y en menor medida gobiernos regionales o municipales. En el caso de empresas consideradas en las investigaciones, estas pertenecen a capitales nacionales como foráneos y asociados al sector minero, forestal, agrícola y energético. En cuanto a la sociedad civil, las entidades consideradas en los análisis son diversas. En primer lugar, destaca el concepto de "comunidad" para denotar el conjunto de personas y organizaciones territoriales directamente implicadas en los proyectos de inversión, destacando comunidades indígenas (mapuche, aymara y quechua). Luego destacan organizaciones funcionales como sindicatos y gremios. Cabe destacar que las organizaciones no gubernamenta-

En alrededor de un tercio de las investigaciones se habla del territorio o territorios indígenas, junto con referirse al conflicto indigena o relacionado con población indígena, en su mayoría del pueblo mapuche. Las especificidades de este corpus merecerían un análisis aparte, que está fuera del alcance de este trabajo. 
les (ONGs) se consignan como parte importante de los casos analizados, pero en ningún trabajo estas organizaciones son el objeto de estudio.

En general, existe en los textos analizados un conjunto de actores que siguen una tendencia clásica de análisis en un sentido de lo que se ha denominado "stakeholders". A excepción del texto de Rodríguez et al. (2020) que desde perspectivas constructivistas amplían el concepto de actor hacia entidades no humanas, asumiendo principios de la "ontología relacional" (Escobar, 2015) o cercanas a la noción de "actor-red" (Latour, 2008) que expande la concepción del ser en cuanto a situaciones históricas y culturalmente definidas. Sin embargo, cabe preguntarse por actores que, si bien escapan a esta ampliación ontológica, podrían considerarse en el plano de los conflictos territoriales en torno a proyectos de inversión. El radio de acción es de carácter global traspasando jurisdicciones nacionales y que suelen tener un "ethos global" (Robinson, 2008; Bartley, 2018). Un buen ejemplo son las organizaciones implicadas en los procesos de certificación privada internacional o aquellas organizaciones involucradas en cadenas globales de valor que, si bien no están necesariamente relacionadas con el proceso de extracción, son parte del proceso de acumulación y legitimación de un proceso productivo.

\section{¿Escalas o niveles?}

En términos de las escalas de investigación, casi la mitad de los trabajos se concentran en una escala local, 8 trabajos en una escala regional, 6 a escala nacional y solo 5 desarrollan un enfoque multiescalar o interescalar. Cabe agregar que, algunas investigaciones se enfocan en escalas más complejas de categorizar y que no responden a elementos constitutivos distintos de límites político-administrativos, más bien se relacionan con límites de uso económico y cultural. Se trata, por ejemplo, de trabajos sobre regiones mineras del norte del país, el territorio forestal del centro-sur de Chile o el territorio indígena mapuche, mencionado como Gulümapu o Wallmapu que comprende varias regiones de la zona sur. Sin embargo, en su gran mayoría, el abordaje de los conflictos socio-ambientales en los artículos del corpus se realiza enfocándose en una sola escala, sea local, regional o nacional, según la disciplina de estudio y el tipo de conflicto y de industria extractiva estudiados. Uno de los principales conflictos abordados, claramente desde una perspectiva interescalar, es el conflicto por el proyecto HidroAysén en la Patagonia, el cual implicaba la construcción de 5 represas y una línea de trasmisión de $2300 \mathrm{~km}$, teniendo de por sí una dimensión interescalar (Silva, 2016). Considerando las características interescalares inherentes al extractivismo, esa dimensión ante todo local y regional de los trabajos publicados se articula con las concepciones de conflicto y de territorio presentadas anteriormente. El enfoque está puesto en la dimensión física del conflicto, así como en las resistencias en el espacio local. Sin embargo, los conflictos socio-ambientales se caracterizan por ser multiescalares, articulando lo local con lo global, pero también una red de territorios (Svampa, 2012).

Más allá de la escala elegida en los trabajos analizados, y en relación con la falta de análisis multi e interescalares, el concepto de escala está también subutilizado en gran parte del corpus. Este concepto, no es sinónimo de nivel político-administrativo, va mucho más allá de un solo contenido espacial: es una construcción geográfica, un conjunto jerárquico de prácticas socio-espaciales. Por eso, autores como Swyngedouw (2004) destacan la contradicción que reside en abordar el mundo desde una escala geográfica dada y fija. Según este mismo autor, las configuraciones escalares son el resultado de procesos socioespaciales que regulan y organizan 
las relaciones de poder. Los actores usan las escalas y juegan con ellas. La escala no es neutra y está en permanente redefinición, por lo cual ofrece pistas de análisis para entender mejor la complejidad socioespacial de los conflictos extractivistas.

\section{Metodología: el predominio del estudio de caso}

Respecto a la metodología, los trabajos revisados presentan poca variedad en cuanto a sus estrategias de investigación. La mayoría se basa en estudios de caso único o múltiples, constituidos por casos subnacionales de comunidades o territorios de influencia de proyectos extractivos.

Cabe destacar que los trabajos comparativos entre macro-regiones o entre países latinoamericanos parecen ser escasos (Heinelt, 2019; Fernández, 2020). Respecto a las técnicas de recogida y análisis de datos, la mayoría de dichos estudios de caso se basan en técnicas cualitativas, tales como observación participante, análisis de contenido de medios y entrevistas. El resto minoritario de los trabajos se dividen entre los que usan técnicas cuantitativas (Rivera et al., 2016; Akchurin, 2020) o mixtas (Romero-Toledo, 2019). Cabe mencionar que sólo uno de los trabajos revisados reconoce fundamentarse en la tradición de la Investigación Acción Participativa (González et al., 2019).

Como lo hemos notado respecto al concepto de extractivismo, existe una convergencia respecto a la estrategia metodológica. Es necesario preguntarse si los estudios críticos respecto a los conflictos territoriales tienen una posición pragmática respecto al uso de técnicas de producción y análisis de datos o más bien una noción de coherencia epistemológica (Bericat, 1998) que emplaza a la elección por un paradigma comprensivo, por sobre una perspectiva explicativa-cuantitativa (Briones, 2002; Ragin, 2007). Sin duda, otros aspectos organizacionales como es la disposición de datos y la presión que se ejerce a conseguir publicaciones, son factores a tener en cuenta.

En resumen, la revisión de estos tres conceptos y tres dimensiones nos permite avanzar hacia la idea de un campo de investigación que tiende a converger, facilitando la creación de una comunidad académica e incluso epistémica. Sin embargo, esta convergencia trae consigo la falta de cuestionamiento de ciertos conceptos centrales como es el de extractivismo. Se percibe más bien como una suma de trabajos al respecto, que como una agregación reflexiva que se abra a interrogar los procesos intensivos en recursos naturales y sus consecuencias situadas en Chile. Por un lado, podemos hablar, en el uso de los conceptos, de una situación en la cual el proceso de conceptualización mismo es dado por hecho, al punto de no ofrecer definiciones precisas de territorio o de extractivismo, conformando un marco teórico unánime que no da lugar a debates. Por otro lado, respecto a las estrategias de investigación, podemos mencionar una reiteración de la metodología de investigación, con la adopción de estrategias similares, que se reproducen en gran parte de los trabajos revisados. Por último, cabe destacar lo valioso de esta primera etapa de publicaciones sobre conflicto, extractivismo y territorio, así como la necesidad de superar lo dado por hecho y la rutina. 


\section{Buscando una nueva etapa}

Los trabajos sobre conflictos extractivo-territoriales han seguido, en parte, al boom minero-energético en el continente, el interés político por la extracción de recursos para financiar políticas de desarrollo, así como a las numerosas protestas y tensiones que estas opciones político-económicas generaron sobre el ambiente y las poblaciones. La primera fase de teorización del extractivismo, junto con el giro eco-territorial, permitió generar un marco teórico conceptual compartido, tanto para investigadores e investigadoras, como para distintos grupos sociales que necesitan instrumentos intelectuales para visibilizar estas situaciones. La segunda fase de estas investigaciones académicas, analizadas para el caso de Chile en esta revisión sistemática, al contrario de la primera de índole más teórica, se orienta hacia la descripción y documentación empírica para entender estos procesos, utilizando el marco conceptual general antes descrito. Este balance de literatura, así como nuestra experiencia colectiva dentro del Coloquio de Conflictos Territoriales de COES, nos permitió identificar los rasgos de rutinización ya señalados. Estos, son a su vez, un punto de partida para pensar una nueva etapa de investigación.

Nuestra propuesta no pretende ser exhaustiva y consiste en una presentación de diferentes pistas a explorar en torno a dos aspectos principales: renovar las metodologías utilizadas y potenciar los conceptos claves fortaleciendo la relación teoría-casos.

\section{Renovación/innovación metodológica}

En materia de metodología, nuestra revisión demuestra que predomina el estudio de caso, donde los casos sirven como estrategia para documentar y describir los conflictos extractivo-territoriales. Sin embargo, el uso reiterado de esta metodología tiende a obviar otra parte clave de la construcción del conocimiento: el análisis. En efecto, numerosos estudios de caso revisados utilizan conceptos a modo de contexto general para la descripción, al contrario de hacer uso de la descripción empírica para nutrir el entendimiento teórico. No hay mucho énfasis en los criterios teóricos de selección de los casos, algo que favorecería su potencial para la investigación.

Para remediar esta tendencia, existen varias estrategias. Primero, se podrían agregar más estudios de caso al conocimiento existente (revisión cautelosa de la literatura sobre casos similares, casos de la misma región o país, casos del mismo sector productivo, etc.). Segundo, se podrían realizar estudios con un mayor número de casos, n pequeño o mediano (Maher, et al., 2019; Campos, 2019), o utilizar métodos cuantitativos (n grande) en vez de monografías. Tercero, invitamos también a comparar de manera más sistemática, ya que los estudios de comparación internacional son un aporte en el estudio de los conflictos extractivo-territoriales (Heinelt, 2019; Fernández, 2020). Las comparaciones permiten poner de relieve las especificidades de los casos, entender el interés empírico específico de una situación, al mismo tiempo que permite entender dinámicas globales, centrales para entender la evolución y el despliegue de los conflictos.

Los estudios comparados se pueden desarrollar de múltiples formas: comparando casos diferentes analizados a escala local; comparando el desarrollo de un conflicto utilizando distintas escalas; comparando distintos casos a nivel internacional, etc. Esto permitiría, también, insistir en la especificidad de la producción académica y del conflicto extractivo-territorial en Chile, para conocer con mayor detalle su relación con la dinámica global y regional latinoamericana. 
¿Cuáles son las características de los conflictos extractivo-territoriales en Chile? ¿En qué medida estas tendencias son similares a otras en Latinoamérica? ¿Cuál es el aporte de las investigaciones sobre y desde Chile al entendimiento del extractivismo en América Latina, al entendimiento de la evolución de los conflictos alrededor del territorio, y a los procesos de construcción social del territorio? Tenemos un acumulado de estudios de casos que dan cuenta de la diversidad del "caso chileno" -gracias a estos últimos años de investigación (afiliación múltiple de los autores y autoras)- sin embargo, para esta nueva etapa, debemos utilizarlos con mayor rigurosidad. Por una parte, se trata de entender lo que hace o no lo específico y particular de las configuraciones extractivas en Chile y, por otra parte, el objetivo es avanzar hacia una mayor conceptualización a partir del estudio empírico. Finalmente, volver a introducir la dinámica global y comparativa permitiría retroalimentar los primeros escritos sobre el tema (Gudynas, Svampa, Acosta), los cuales proponían teorías de mayor alcance.

\section{Potenciar los conceptos claves y fortalecer la relación teoría-casos}

Después de una primera etapa de investigación fuertemente teórica, identificamos una etapa de mayor documentación empírica y acumulación de estudios descriptivos. La tercera etapa de investigación podría, a nuestro modo de ver, fortalecer la relación teoría-casos. Ante todo, cabe señalar que este campo de estudio, caracterizado por su multidisciplinariedad hace que algunas diferencias conceptuales no sean completamente salvables -tampoco sería deseable una unificación conceptual completa. Sin embargo, un camino importante es volver sistemáticamente al origen de los conceptos, y prestar atención a su densidad. En efecto, el uso de conceptos como conflicto, extractivismo y territorio es a menudo demasiado liviano (incluso en las investigaciones de algunos de los autores del presente estudio). Son conceptos que tienen una densidad importante, que se empobrecen cuando se usan como etiquetas. Podríamos fortalecer más el vínculo entre la observación empírica y el refinamiento conceptual.

Por ejemplo, el uso del concepto conflicto como expresión de una tensión o confrontación entre dos partes elude una concepción más latente del conflicto o no dicotómicas del conflicto, como puede ser el caso en situaciones donde existe un claro desequilibrio de poder entre los actores. Los matices esconden a veces otras formas de dominación para sujetos menos empoderados o que no tienen recursos políticos, económicos, sociales o referencias valóricas suficientes. Un uso diferente del concepto conflicto podría ser pertinente en Chile donde el conflicto se ha manifestado de forma más latente en comparación con otros países de la región (vínculo con la especificidad de cada país). Tal vez estudiar lo que no es un "caso" mediático, político, visible, donde hay movilización social, permitiría justamente desenfocar el lente de estudio sobre la acción colectiva visible y entender lo que pasa cuando no hay aparentemente conflictividad. Esto implica, por un lado, repensar estrategias de investigación, identificando los límites de uso del estudio de caso, de las bases cuantitativas basadas en prensa o el rastreo de organismos públicos o no gubernamentales (Instituto Nacional de Derechos Humanos -INDH-, Observatorio de Conflictos Mineros en América Latina -OCMAL- y el Mapa de Justicia Ambiental -EJATLAS- por citar las bases más usadas en Chile). Sin embargo, se trata también de redefinir lo que entendemos por una investigación comprometida con develar las relaciones de poder. Estas se han concentrado en acompañar, clarificar y co-construir lo expuesto por actores sociales considerados como en "resistencia". La etapa siguiente podría seguir con esta dinámica comprometida, arraigada en la ecología política, pero esta vez para estudiar con mayor detalle procesos, actores, y dinámicas que permitan entender la continuidad de la dominación capitalista. 
Otro ejemplo para sobrepasar la rutinización está relacionado con el uso del concepto "territorio". La literatura permitió insistir en el territorio como construcción social y demostrar cómo el conflicto alrededor de recursos naturales conlleva distintos grupos sociales a reflexionar sobre su territorialidad y su relación con el espacio vivido. Esta conceptualización surge de un análisis empírico centrado en estudios de casos, en su mayoría enfocados en el actor territorial o territorializado: la "comunidad" (también un concepto rutinizado), pobladores, grupos étnicos, habitantes, etc. Sin embargo, las investigaciones empíricas describen la presencia de otros actores como son las empresas y el Estado: ¿Cuál sería el territorio de las empresas y según las empresas? ¿Y el territorio del Estado y según el Estado en estos casos? Fortalecer la relación entre lo empírico y la teoría permite seguir indagando sobre el concepto de territorio como desvelador de distintas contradicciones, las cuales producen conflicto.

En cuanto al concepto de extractivismo, usado a menudo como elemento descriptivo contextual, varios trabajos aportan una precisión sectorial: "extractivismo minero" "agroindustrial", "energético", "pesquero", "forestal". Existe en Chile una discusión sobre la inclusión o no de ciertos sectores productivos en el marco conceptual del extractivismo (Pino \& Carrasco, 2019). Podríamos aprovechar estas preguntas para nutrir el concepto: ¿Cuál es la dinámica específica de lo minero o de lo forestal? ¿Qué hay en común? ¿Cuál es la particularidad de un sector en relación con otro? ¿Qué los diferencia respecto a la dicotomía renovable/no renovable? Por ejemplo, para estudiar minería se deben estudiar lugares de extracción, lugares de refinamiento del material, sitios de exportación y espacios que sirven de almacenamiento de desechos. Otras preguntas que surgen son los impactos similares o distintos de cada sector sobre la producción de territorio, sobre la configuración del conflicto, sobre la organización de los actores y sus redes políticas nacionales. El punto es especialmente relevante en Chile, dada la diversificación productiva de la actividad extractiva de recursos naturales a gran escala. Por último muchos trabajos empiezan, también, su reflexión en el periodo contemporáneo, y podríamos seguir indagando sobre el concepto de extractivismo con una perspectiva más sociohistórica, tanto para dar cuenta de su arraigo o vinculación con períodos anteriores (como ocurre con los ciclos mineros), como también para investigar los nuevos fenómenos, en su relación con ciclos anteriores (como ocurre por ejemplo en Chiloé, que tuvo una fuerte producción forestal antes del actual predominio de la salmonicultura; o con el golfo de Arauco, donde el siglo XIX se fundía gran parte del cobre chileno, luego se forestó para tener madera para la minería $y$, actualmente se ha expandido la forestación para la producción y exportación de celulosa).

Los comentarios anteriores toman mayor importancia cuando se trata de poner en relación estos conceptos, como es el caso de los estudios que analizamos, es decir, cuando se vincula conflicto y territorio, extractivismo y territorio, y conflicto con extractivismo. Aquí se evidencia la necesidad de distinguir los términos adecuadamente para poder relacionarnos de manera productiva. Si el extractivismo es el nuevo modelo de acumulación que impone sus reglas, ello ocurre sea cual sea el territorio y define un conflicto per se, antes de la aparición de los actores específicos. Las variaciones serán un asunto más bien episódico. En el extremo, la relación entre la conceptualización general y estructural del extractivismo y los casos consiste en "develar" o poner en evidencia lo que ya la teoría había predicho (que el extractivismo despoja los territorios y que se producen resistencias que tienen significado político, puesto que se refieren al modelo de acumulación). Pero ¿es esa la tarea de la investigación de casos específicos? En este tipo de estudios parecería más productivo el utilizar el fenómeno general (extractivismo) como un "telón 
de fondo" que ayuda a entender rasgos comunes, pero la tarea de la investigación territorial o del conflicto tiene aún todo el campo por explorar (a diferencia de una investigación "estructuralista" de cómo evoluciona el modelo).

Por otra parte, es necesario distinguir el conflicto como acción colectiva del conflicto extractivo a secas. La constatación más evidente es que no en todos los contextos extractivos surge la acción colectiva, que la acción colectiva no es igual en todos los territorios y que, por último, las consecuencias de la acción colectiva son muy diversas también (según su dinámica, según la gestión política, según el tipo de territorio, etc.). Todas esas son variables relevantes de desarrollar y analizar en este campo de investigación.

Por último, la idea predominante de "territorio como construcción social" obliga a mirar hacia la constitución de actores, sus dinámicas conflictivas (o no) y los territorios que de allí emergen y evolucionan. Identificar conflicto con "resistencia" no ayuda en esa dirección. Pero sobre todo el territorio como variable obliga a considerar con mayor profundidad "los extractivismos", puesto que la materialidad de la producción, las relaciones de trabajo, las externalidades ambientales, la demografía, entre otras, son dimensiones que varían según sectores, condiciones tecnológicas e, incluso, con cambios en los mercados (mayor o menor intensidad de la producción).

\section{Conclusión}

Nuestro propósito con este artículo ha sido contribuir a la discusión académica sobre conflictos territoriales y extractivismo en Chile. El análisis presentado, sobre este corpus de 32 artículos, permite visibilizar la forma en la cual se está dando la discusión académica y sirve de base para formular algunas propuestas que esperamos sean de interés para la comunidad que comparte la preocupación por el extractivismo y sus consecuencias.

En este sentido, nuestro esfuerzo se inscribe en la conformación de una comunidad académica multinivel e interdisciplinaria. El componente multinivel implica considerar las dinámicas subnacionales primordiales, pero en conexión con discusiones que tienen inherentemente un carácter global. Mientras, lo interdisciplinario busca hacerse cargo de la complejidad de los fenómenos abordados. Los límites que detectamos en el uso de los conceptos de escala o territorio tienen que ver en buena parte con que no se han hecho transversales todavía algunos avances de la geografía contemporánea. Lo mismo pasa desde otras disciplinas para la aproximación a los actores, sus configuraciones y estrategias. Se requiere, entonces, tomar el tiempo de la interdisciplina y la conexión multinivel, lo que no es un desafío menor en la academia contemporánea.

La presente revisión nos ha permitido pensar y proyectar nuevos rumbos sobre las investigaciones futuras. Por ejemplo, considerar regiones que no han recibido atención académica (bajo los criterios de esta revisión) y donde, actualmente, se están levantando grandes proyectos extractivistas. O, más allá de proyectos particulares, reconsiderar discusiones como las fronteras entre lo urbano y rural al pensar el extractivismo. En un momento histórico, de varias encrucijadas, a nivel nacional con el proceso constituyente, e internacional, con el nuevo superciclo de materias primas que se perfila, esperamos que este artículo, y las discusiones que pueda suscitar, contribuya a la consolidación del espacio de discusión académica sobre estas materias. 


\section{Agradecimientos}

Los autores agradecen el apoyo del Centro de Estudios de Conflicto y Cohesión Social COES (ANID/FONDAP/15130009), de Fondecyt (ANID /FONDECYT/1190070; ANID /FONDECYT/1180496; ANID /FONDECYT/ 11181188; ANID /FONDECYT/3190686), del Centro de Ciencia del Clima y la Resiliencia CR2 (ANID/FONDAP/15110009) y del Centro Nacional de Investigación para la Gestión Integrada de Desastres Naturales (ANID/FONDAP/15110017).

\section{Referencias}

AKCHURIN, M. Mining and Defensive Mobilization Explaining Opposition to Extractive Industries in Chile. Sociology of Development, 2020, Vol. 6, No 1, p. 1-29. doi: https://doi.org/10.1525/ sod.2020.6.1.1

ALIMONDA, H., TORO, C. \& MARTÍN, F. (Coord.) Ecología política latinoamericana: pensamiento crítico, diferencia latinoamericana y rearticulación epistémica. $1^{\circ}$ ed. Buenos Aires: CLACSO, 2017. Disponible en Internet: http://biblioteca.clacso.edu.ar/clacso/gt/20171030111951/GT_Ecologia_politica_Tomo_I.pdf

ARBOLEDA, M. Planetary Mine: Territories of Extraction Under Late Capitalism. $1^{\circ}$ ed. New York: Verso, 2020.

ARSEL, M. HOGENBOOM, B. \& PELLEGRINI, L. The extractive imperative in Latin America. The Extractive Industries and Society, 2016, N³, p. 880-887. doi: https://doi.org/10.1016/j.exis.2016.10.013

BARTLEY, T. Transnational Corporations and Global Governance. Annual Review of Sociology, 2018, No 44, p. 145-165. doi: https://doi.org/10.1146/annurev-soc-060116-053540

BEBBINGTON, A. Latin America: contesting extraction, producing geographies. Singapore Journal of Tropical Geography, 2009, Vol. 30, № 1, p. 7-12. doi: https://doi.org/10.1111/j.14679493.2008.00349.x

BEBBINGTON, A. \& BURY, J. (Eds.) Subterranean Struggles: New Dynamics of Mining, Oil and Gas in Latin America. Austin: University of Texas Press, 2014.

BERICAT, E. La integración de los métodos cuantitativo y cualitativo en la investigación social. Significado y medida. $1^{\circ}$ ed. Barcelona: Editorial Ariel, 1998. Disponible en internet: http://mastor. $\mathrm{cl} / \mathrm{blog} / \mathrm{wp}$-content/uploads/2019/07/Bericat-La-Integracion-de-Los-Metodos-Cuanti-y-Cuali.pdf

BERGER, P.L. \& LUCKMANN, T. The Social Construction of Reality. A Treatise in the Sociology of Knowledge. Garden City, NY: Double Day. 1966.

BOLADOS, P. Conflictos socio-ambientales/territoriales y el surgimiento de identidades post neoliberales (Valparaíso-Chile). Izquierdas, 2016, No 31, p.102-129. doi: https://dx.doi.org/10.4067/ S0718-50492016000600102 
BOLADOS, P., HENRIQUEZ, F., CERUTI, C \& SANCHEZ, A. La eco-geo-política del agua: una propuesta desde los territorios en las luchas por la recuperación del agua en la provincia de Petorca (Zona central de Chile). Revista Rupturas, 2017, Vol. 8, N¹, p. 159-191. doi: https://doi.org/10.22458/ rr.v8i1.1977

BOLADOS, P. \& BOCCARA, G. Du néolibéralisme multiculturel aux mobilisations post-multicultureIles. Actuel Marx, 2015, Vol. 2, Nº 56, p. 74-84. doi: https://doi.org/10.3917/amx.056.0074

BOLADOS, P. \& SANCHEZ, A. Una ecología política feminista en construcción: El caso de las "Mujeres de zonas de sacrificio en resistencia", Región de Valparaíso, Chile. Psicoperspectivas, 2017, Vol. 16, №2, p. 33-42. doi: http://dx.doi.org/10.5027/psicoperspectivas-vol16-issue2-fulltext-977

BRIONES, G. Epistemología de las Ciencias Sociales. $1^{\circ}$ ed. Bogotá: Arfo Editores, 2002. Disponible en Internet: http://cordescorporacion.cl/wp-content/uploads/2018/03/epistemologi\%CC\%81a-briones.pdf

BUSTOS, B. \& PRIETO, M. Ecología Política en (desde y por) Chile: Posibilidades, desafíos u contribuciones. En NÚÑEZ, A., ALISTE, E. y MOLINA, R. (Eds.) (Las) Otras Geografías en Chile. Perspectivas sociales y enfoques críticos. Santiago de Chile: LOM, 2019, p. 85-104.

BUSTOS, B., PRIETO, M., \& BARTON, J. Ecología política en Chile: Naturaleza, propiedad, conocimiento y poder. Santiago de Chile: Editorial Universitaria, 2015.

CAMPOS, F. Ecological modernization from the actor's perspective: Spatio-temporality in the narratives about socio-ecological conflicts in Chile. Time \& Society, 2019, Vol. 8, No 3, p. 1239-1271. doi: https://doi.org/10.1177/0961463X17752284

CARDOSO, F. \& FALETTO, E. Dependencia y desarrollo en América Latina. Ciudad de México: Siglo XXI Editores,1969.

CUEVAS, H. \& BUDROVICH, J. La neoliberalización de los puertos en Chile: el caso de la ciudad-puerto de Valparaíso. Revista Austral de Ciencias Sociales, 2020, №38, p. 337-363. doi: https://doi.org/10.4206/rev.austral.cienc.soc.2020.n38-17

ESCOBAR, A. Territories of Difference: place, movements, life, redes. Durham: Duke University Press, 2008.

ESCOBAR, A. Territorios de diferencia: la ontología política de los "derechos al territorio". Cuadernos de Antropología Social, 2015, N 41, p. 25-38. doi: https://doi.org/10.34096/cas.i41.1594

FERNÁNDEZ, J. El territorio como espacio contradictorio: promesas y conflictos en torno a la actividad extractiva en Ecuador, Colombia, Perú y Chile. Revista Latinoamericana de Estudios Urbano Regionales, 2020, Vol. 46, No 137, p. 225-246. doi: http://dx.doi.org/10.4067/S025071612020000100225 
GONZÁLEZ, M. \& ZOGRAFOS, C. How sovereignty claims and "negative" emotions influence the process of subject-making: Evidence from a case of conflict over tree plantations from Southern, Chile. Geoforum, 2017, № 78, p. 61-73. doi: http://dx.doi.org/10.1016/j.geoforum.2016.11.012

GONZÁLEZ, M., LÓPEZ, S. \& PACHECO, S. El sentipensar extractivo colonial: Geografías emocionales de la extracción en Gülumapu, el territorio mapuche en el sur de Chile. Journal of Latin American Geography, 2019, Vol. 18, № 3, p. 85-109. doi: http://doi.org/10.1353/lag.2019.0055

GUDYNAS, E. La ecología política del progresismo sudamericano: los límites del progreso y la renovación verde de la izquierda. Sin Permiso, 2010, № 8, p. 147-167. Disponible en Internet: https:// www.researchgate.net/publication/277220330_La_ecologia_politica_del_progresismo_sudamericano_los_limites_del_progreso_y_la_renovacion_verde_de_la_izquierda

HEINELT, S. How to face the "fight of an ant against a giant"? Mobilization capacity and strategic bargaining in local ethnic conflicts in Latin America. Zeitschrift für Vergleichende Politikwissenschaft, 2019, №13, p. 93-133. doi: https://doi.org/10.1007/s12286-019-00417-5

KRIPPRENDOFF, K. Content Analysis. An Introduction to its Methodology. United States of America: SAGE Publications Inc., 2003.

LATOUR, B. Reensamblar lo social. Buenos Aires: Ediciones Manantial, 2008.

MAHER, R., VALENZUELA, F. \& BOHM, S. The Enduring State: An analysis of governance-making in three mining conflicts. Organization Studies, 2019, Vol. 40, №8, p. 1169-1191. doi: https://doi. org/10.1177/0170840619847724

MANCILLA, N. Gestionando el neoextractivismo en un conflicto ambiental en el sur de Chile. Chasqui. Revista Latinoamericana de Comunicación, 2017, Nº136, p. 195-211. doi: https://doi. org/10.16921/chasqui.v0i136.3316

MARTÍNEZ-ALIER, J. Ecología política del extractivismo y justicia socio-ambiental. Interdisciplina, 2015, Vol. 3, No 7, p. 53-73. doi: http://dx.doi.org/10.22201/ceiich.24485705e.2015.7.52384

OCAMPO, A., GIRONAS, J. \& VALLS, A. A rule-based approach for preventive identification of potential conflictive criteria in mining operations in Chile. Journal of Cleaner Production, 2018, Vol. 184, No 20, p. 559-568. doi: https://doi.org/10.1016/j.jclepro.2018.02.202

PALMA, K. \& ALCAINO, C. Mining the Media: How Community Radio Breaks Though Extractivist Discourse Articulations in a Context of Disaster and Socio-environmental Conflicts. Environmental Communication, 2020, Vol. 14, Nº6, p. 830-843. doi: https://doi.org/10.1080/17524032.2020.1756 887

PINO, A. \& CARRASCO, N. Extractivismo forestal en la comuna de Arauco (Chile): internalización y formas de resistencia. Revista Colombiana de Sociología, 2019, Vol. 42, N¹, p. 207-226. doi: https://doi.org/10.15446/rcs.v42n1.73233 
PORTO-GONÇALVES, C. De Saberes y de Territorios: diversidad y emancipación a partir de la experiencia latino-americana. Polis, 2009, Vol. 8, No22, p. 121-136. Disponible en Internet: https:// scielo.conicyt.cl/pdf/polis/v8n22/art08.pdf

PORTO-GONÇALVES, C. \& LEFF, E. Political Ecology in Latin America: The Social Re-Appropiation of Nature, the Reinvention of Territories and the Construction of an Environmental Rationality. Desenvolv. Meio Ambiente, 2015, Vol. 35, p. 65-88. doi: http://dx.doi.org/10.5380/dma.v35i0.43543

PREBISCH, R. Crecimiento, desequilibrio y disparidades: interpretación del proceso de desarrollo económico. Estudio económico de América Latina y el Caribe, Naciones Unidas Comisión Económica para América Latina y el Caribe (CEPAL), 1950, № 1110. Disponible en: https://repositorio. cepal.org/bitstream/handle/11362/1110/006_es.pdf?sequence=1\&isAllowed=y

RAGIN, C. Introduction. En RAGIN, C. \& BECKER, H. (Eds.) What is a case? Exploring the Foundations of Social Inquiry. New York: Cambridge University Press. 1992.

RAGIN, C. La construcción de la investigación social: Introducción a los métodos y su diversidad. Bogotá: Siglo del hombre Editores, 2007. Disponible en Internet: http://metodos-avanzados.sociales.uba.ar/wp-content/uploads/sites/216/2014/04/Investigacion_ragin.pdf

REYES, S. \& RODRIGUEZ, J. Proyecto Hidroaysén: capitalismo extractivista, regulación estatal y acción colectiva en la Patagonia. Polis, 2015, Vol. 14, N40, p. 439-467. doi: http://dx.doi.org/10.4067/ S0718-65682015000100021

RIVERA, D., GODOY, A., LILLO, M., ALVEZ, A., DELGADO, V., GONZALO, C., MENASALVAS, E., COSTUMERO, R. \& GARCIA, A. Legal disputes as a proxy for regional conflicts over water rights in Chile. Journal of Hydrology, 2016, N535, p. 36-45. doi: https://doi.org/10.1016/j.jhydrol.2016.01.057

ROBINSON, W. Latin America and Global Capitalism. Baltimore: John Hopkins University Press, 2008.

RODRIGUEZ, J., ORTIZ, C. \& BROITMAN, C. Chile, país minero. Licencia social y lugares de enunciación en los conflictos socioambientales en Chile. Izquierdas, 2020, Vol. 49, p. 2900-2922.

ROMERO-TOLEDO, H. Extractivismo en Chile: la producción del territorio minero y las luchas del pueblo aimara en el Norte Grande. Colombia Internacional, 2019, № 98, p. 3-30. doi: https://doi. org/10.7440.colombiaint98.2019.01

ROMERO-TOLEDO, H. \& SAMBOLIN, A. Indigeneidad y territorio: los Aymaras y Qechuas en el norte de Chile. Scripta Nova. Revista Electrónica de Geografía y Ciencias Sociales, 2019, Vol. 23, No 611, p. 1-32. doi: https://doi.org/10.1344/sn2019.23.20408

SANTIBAÑEZ, H. Conflictos socio-ambientales en el área metropolitana de Valparaíso. Revista Austral de Ciencias Sociales, 2018, №35, p. 261-281. doi: https://doi.org/10.4206/rev.austral.cienc. soc.2018.n35-15 
SILVA, E. Patagonia without Dams! Lessons of a David vs. Goliath campaign. The Extractive Industries and Society, 2016, Vol. 3, N4, p. 947-957. doi: https://doi.org/10.1016/j.exis.2016.10.004

SKILTON, P. A comparative study of communal practice: Assessing the effects of taken-for-granted-ness on citation practice in scientific communities. Scienometrics, 2006, № 68, p. 73-96. doi: https://doi.org/10.1007/s11192-006-0084-2

SVAMPA, M. Consenso de los Commodities, Giro Ecoterritorial y pensamiento crítico en América Latina. En SADER, E. y GENTILI, P. (Eds.) Movimientos Socioambientales en América Latina. Buenos Aires: CLACSO, 2012. Disponible en Internet: http://biblioteca.clacso.edu.ar/clacso/ osal/20120927103642/OSAL32.pdf

SVAMPA, M. Las fronteras del neoextractivismo en América Latina. Conflictos socioambientales, giro ecoterritorial y nuevas dependencias. $1^{\circ}$ ed. Wetzlar: CALAS, 2019. Disponible en Internet: http://calas.lat/sites/default/files/svampa_neoextractivismo.pdf

SVAMPA, M. \& STEFANONI, P. (Comp.) Bolivia: memoria, insurgencia y movimientos sociales. Buenos Aires: CLACSO-Editorial El Colectivo, 2007. Disponible en Internet: http://biblioteca.clacso. edu.ar/clacso/osal/20120511050634/bolivia.pdf

SWYNGEDOUW, E. Scaled Geographies: Nature, Place and the Politics of Scale. En SHEPPARD, E. \& McMASTER, R. (Eds.) Scale and Geographic Inquiry: Nature, Society and Method. New Jersey: John Wiley \& Sons, 2004, p. 129-153. doi : https://doi.org/10.1002/9780470999141.ch7

WEBER, R. Basic Content Analysis. United States of America: SAGE Publications, Inc., 1990.

YIN, R. Case Study Research. Design and Methods. United States of America: SAGE Publications, Inc., 2009. 